\title{
Steam Methane Reformation Testing for Air-Independent Solid Oxide Fuel Cell Systems
}

Kamwana N. Mwara

NASA Johnson Space Center

Fuel Cell Seminar 2015

Recently, NASA has been looking into utilizing landers that can be propelled by $\mathrm{LOX}-\mathrm{CH}_{4}$, to be used for long duration missions. Using landers that utilize such propellants, also provides the opportunity to use solid oxide fuel cells as a power option, especially since they are able to process methane into a reactant through fuel reformation. One type of reformation, called steam methane reformation, is a process to reform methane into a hydrogen-rich product by reacting methane and steam (fuel cell exhaust) over a catalyst. A steam methane reformation system could potentially use the fuel cell's own exhaust to create a reactant stream that is hydrogen-rich, and requires less internal reforming of the incoming methane. Also, steam reformation may hold some advantages over other types of reforming, such as partial oxidation (PROX) reformation. Steam reformation does not require oxygen, while up to $25 \%$ can be lost in PROX reformation due to unusable CO2 reformation. NASA's Johnson Space Center has conducted various phases of steam methane reformation testing, as a viable solution for in-space reformation. This has included using two different types of catalysts, developing a custom reformer, and optimizing the test system to find the optimal performance parameters and operating conditions. 


\section{Steam Methane Reformation Testing for Air-Independent Solid Oxide Fuel Cell Systems}

2015 Fuel Cell Seminar

Kamwana N. Mwara/NASA JSC 


\section{Fuel Cells at NASA}

- Gemini, Apollo, and Space Shuttle used fuel cells as main power source for vehicle and water source for life support and thermal

PEM (Gemini) and Alkaline (Apollo, Shuttle) fuel cells were used

Ideal for short (less than 3 weeks) missions when the complete mission load of $\mathrm{O}_{2}$ and $\mathrm{H}_{2}$ can be launched with the vehicle

- New missions that might require long-duration stays in orbit or at a habitat, cannot rely on the availability of pure reactants and should aim to be sun-independent - a problem for which Solid Oxide Fuel Cells might be the answer 


\section{$\mathrm{LOX} / \mathrm{LH} 2$ vs. $\mathrm{LOX} / \mathrm{CH}_{4}$ Usage}

- $\quad$ Recently, NASA has investigated \& developed LOX/CH4-propelled landers (e.g. Morpheus). In order to preserve mission flexibility, fuel cells should be studied as a potential power source.

- Previous work at JSC has identified the volumetric and mass benefits of $\mathrm{LOX} / \mathrm{CH}_{4}$ propelled vehicles vs $\mathrm{LH}_{2} / \mathrm{LO}_{2}$

- The availability of $\mathrm{LOx} / \mathrm{CH}_{4}$ introduces solid oxide fuel cells (SOFCs) as an option, due to their ability to efficiently utilize those reactants.

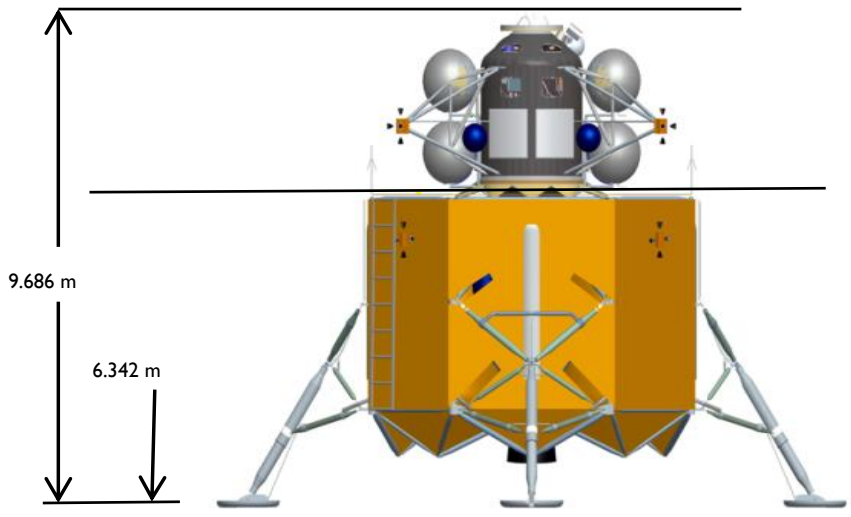

LH2/LO2 Lander Size

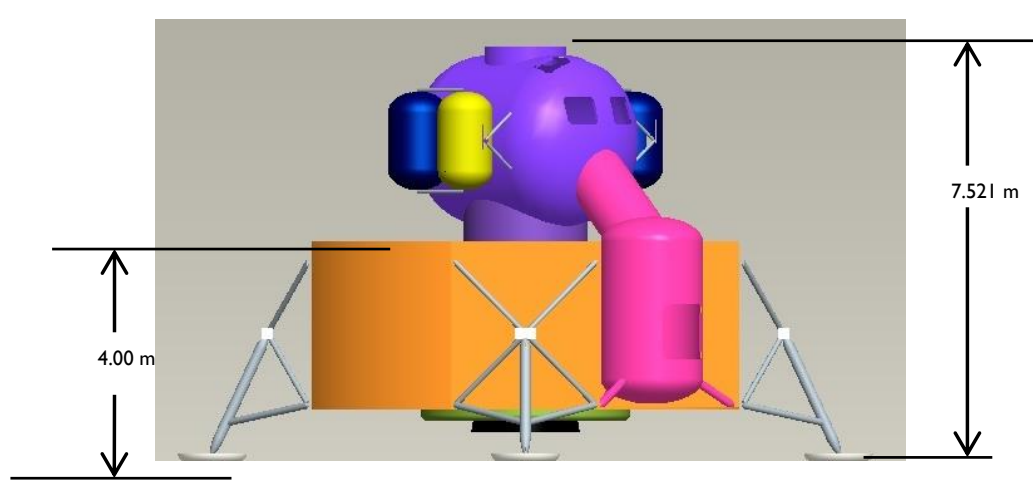

LOX/Methane Lander Size 


\section{Steam Reforming Introduction}

- SOFCs allow internal reforming to convert $\mathrm{CH}_{4}$ into $\mathrm{H}_{2}$ for fuel

- Some external reforming of the fuel stream is optimal

- Utilizing an external steam methane reformer (SMR) would be the first step to creating a more efficient SOFC system
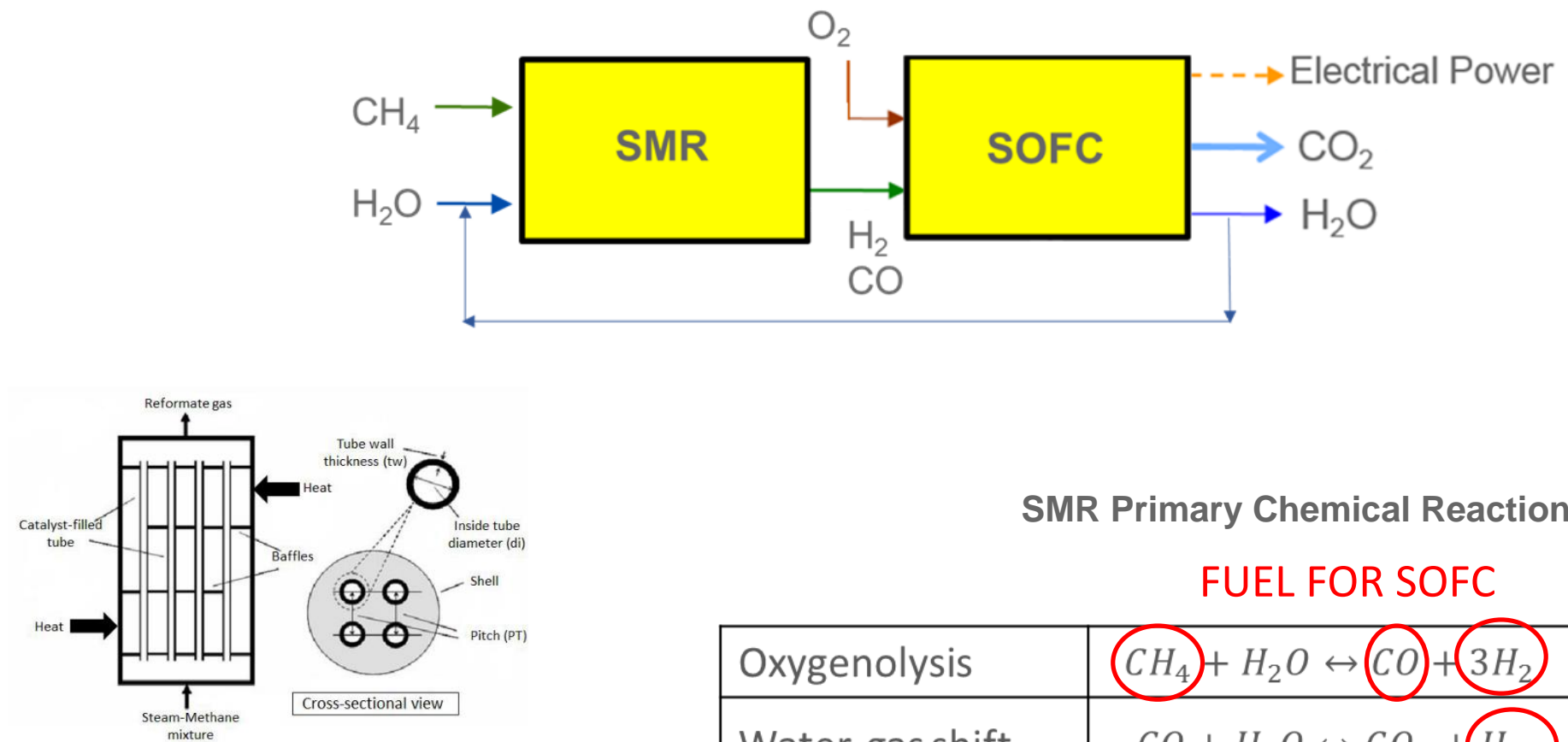

SMR Primary Chemical Reactions

FUEL FOR SOFC

\begin{tabular}{|l|c|l|}
\hline Oxygenolysis & $\left(\mathrm{CH}_{4}\right)+\mathrm{H}_{2} \mathrm{O} \leftrightarrow \mathrm{CO}+\mathrm{BH}_{2}$ & $\Delta \mathrm{H}_{r \times n}^{o}=206 \mathrm{~kJ} / \mathrm{mol}$ \\
\hline Water-gas shift & $\mathrm{CO}+\mathrm{H}_{2} \mathrm{O} \leftrightarrow \mathrm{CO}_{2}+\mathrm{H}_{2}$ & $\Delta \mathrm{H}_{r x n}^{o}=-41 \mathrm{~kJ} / \mathrm{mol}$ \\
\hline
\end{tabular}




\section{SMR - Phase 1}

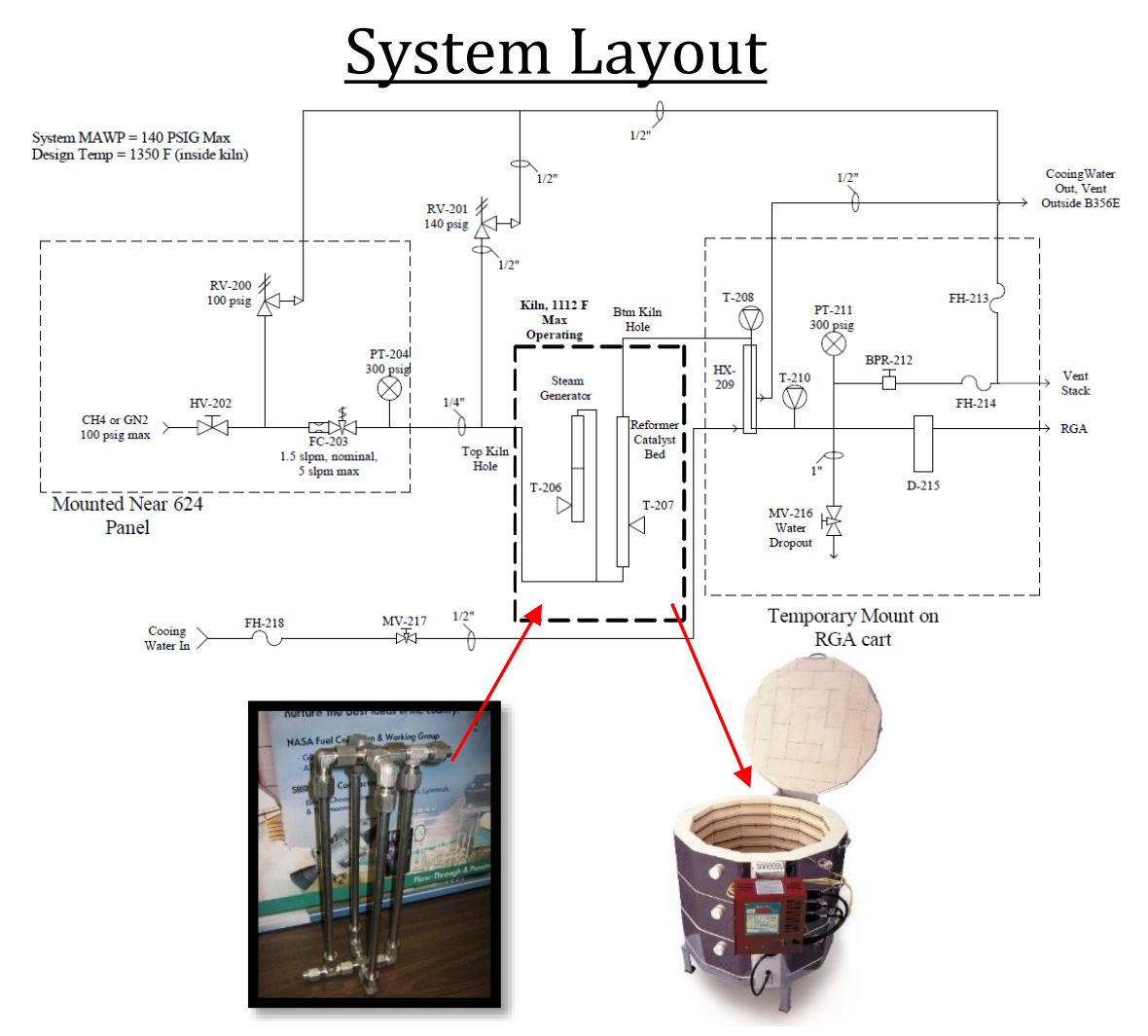

\section{Catalyst}

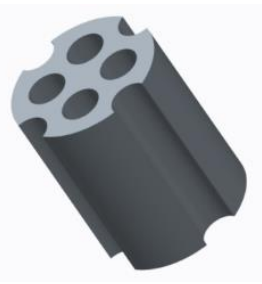

Nickel Oxide Catalyst

Reasons for selection:

- Low cost

- Reasonably good efficiency

\begin{tabular}{|c|c|c|c|c|c|}
\hline \multicolumn{6}{|c|}{ Hot Flow Test (With Preheating of Gases) } \\
\hline Test \# & $\begin{array}{c}\mathrm{CH}_{4} \text { Mass } \\
\text { Flow Rate } \\
{[\mathrm{g} / \mathrm{min}]}\end{array}$ & $\begin{array}{c}\mathrm{H}_{2} \mathrm{O} \text { Mass Flow } \\
\text { Rate } \\
{[\mathrm{g} / \mathrm{min}]}\end{array}$ & $\begin{array}{c}\text { Steam to } \\
\text { Methane } \\
\text { Ratio } \\
{[\mathrm{mol} / \mathrm{mol}]}\end{array}$ & $\begin{array}{c}\text { SMR } \\
\text { Temperature } \\
{\left[{ }^{\circ} \mathrm{F}\right]}\end{array}$ & $\begin{array}{c}\text { System } \\
\text { Pressure } \\
{[\mathrm{psia}]}\end{array}$ \\
\hline 1 & 0.982 & 1.2 & 1 & 1020 & 14.7 \\
\hline 2 & 0.982 & 2.3 & 2 & 1020 & 14.7 \\
\hline 3 & 0.982 & 3.3 & 3 & 1020 & 14.7 \\
\hline
\end{tabular}




\section{SMR - Phase 1}

Generated $\mathrm{H}_{2}$ production, and also high build-up of upstream pressure

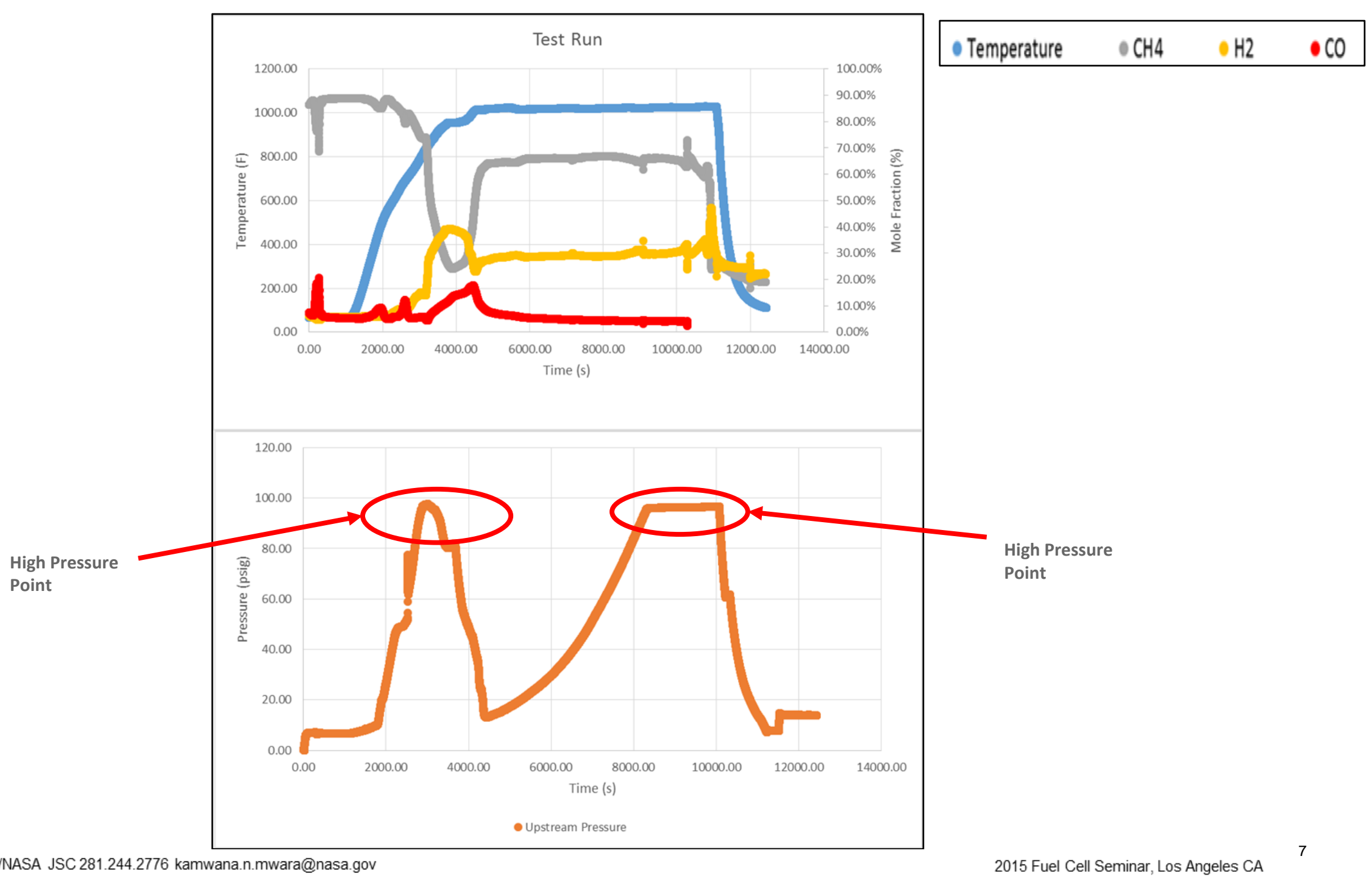




\section{SMR - Phase 1}

Test results:

- Initial production of Hydrogen

- Carbon deposition blockage in the catalyst bed

\section{Conclusions:}

- Use of a kiln to produce steam

- No steam flow regulation (steam-to-methane ratio)

- Catalyst was not reduced:

$$
\mathrm{NiO}+\mathrm{H}_{2} \leftrightarrow \mathrm{Ni}+\mathrm{H}_{2} \mathrm{O}
$$

\section{Phase 2 recommendations:}

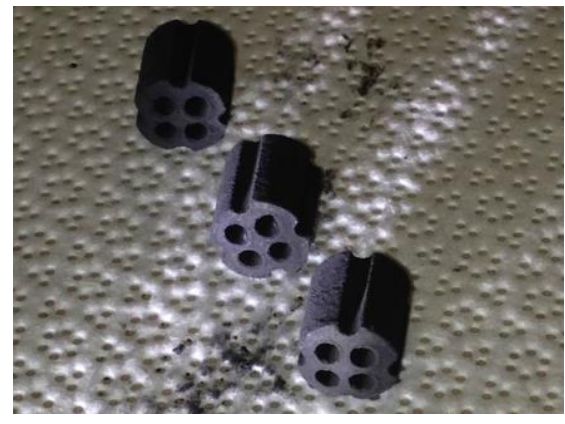

- Use syringe pumps to regulate water flow

- Use a separate heater to generate steam

- Reduce catalyst 


\section{SMR - Phase 2}

\section{Objective:}

- Determine optimal operating conditions, for 1 cylinder SMR reactor system design

- Based on updated theoretical Matlab model

- Incorporate recommendations from Phase 1 test results

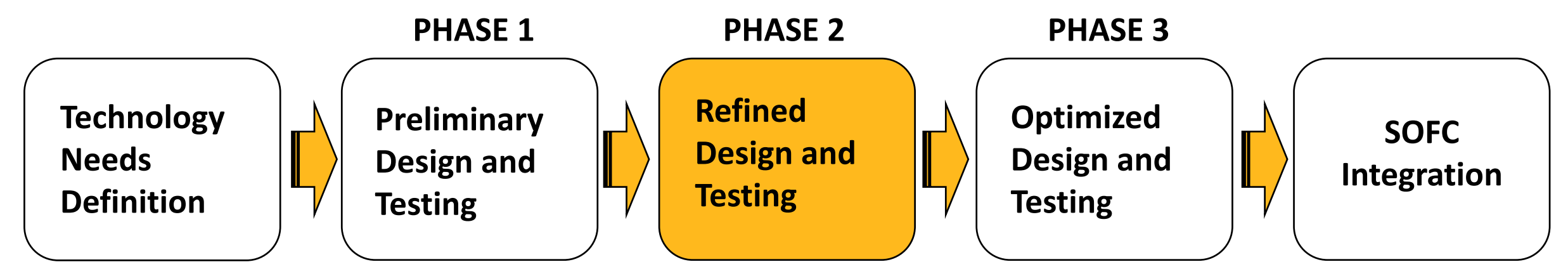




\section{SMR - Phase 2}

\section{System Layout}

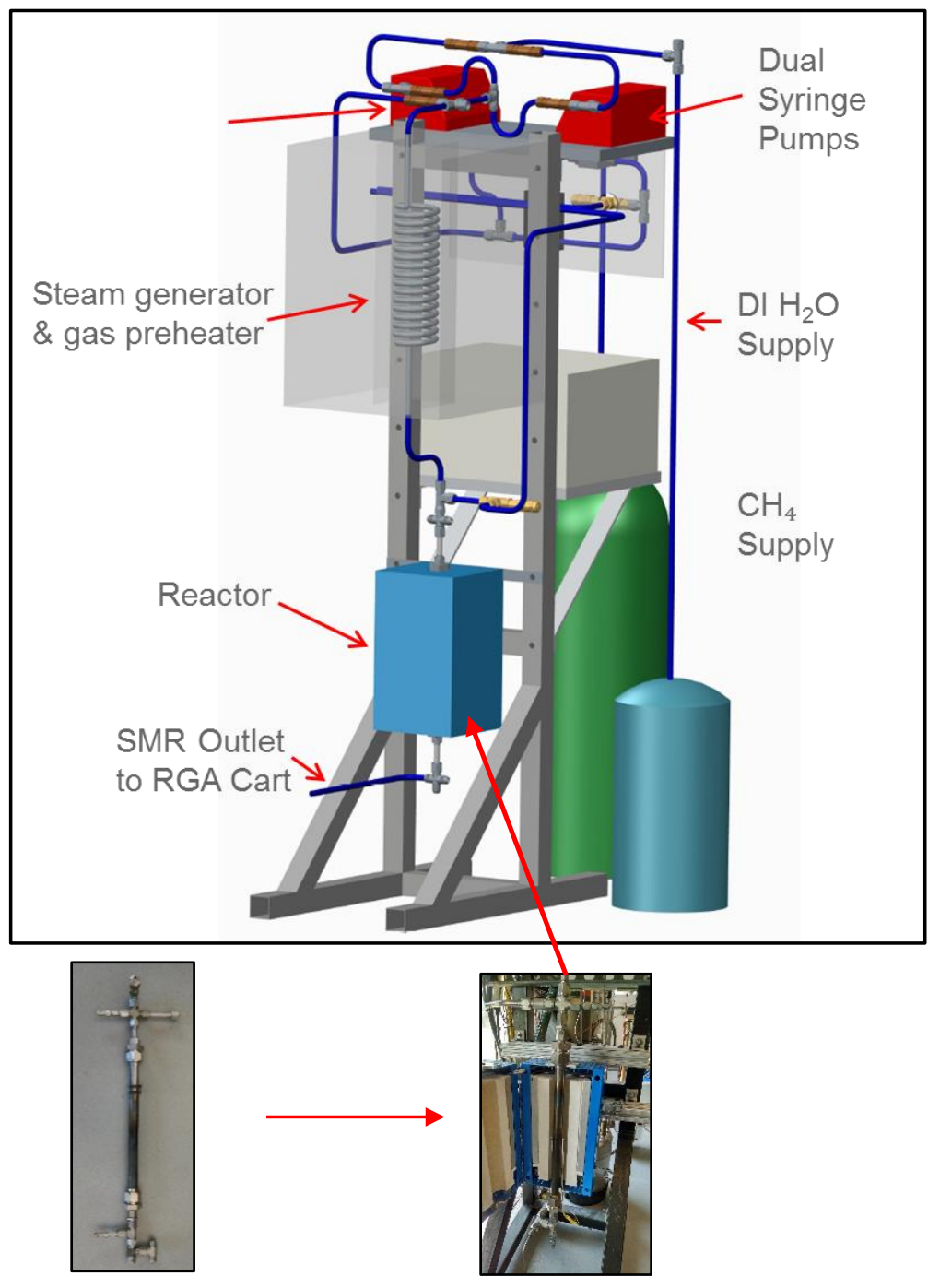

\section{$\underline{\text { Improvements }}$}

\section{Before \\ Passive Water Control \\ - Tubes filled with $\mathrm{H}_{2} \mathrm{O}$ and heated; no control over amount of steam generation and delivery}

Steam generator \& reformer placed in kiln

Catalyst was not reduced

Four tube reactor design

\section{After}

Active water control

- Syringe pumps deliver specific flow rate of $\mathrm{H}_{2} \mathrm{O}$ to steam generator

Separate tube furnaces for steam generator and reformer

- Better heating control

Catalyst reduced by hydrogen, before test runs

One tube reactor design

\begin{tabular}{|c|c|c|c|c|c|}
\hline \multicolumn{7}{|c|}{ Hot Flow Test (With Preheating of Gases) } \\
\hline Test \# & $\begin{array}{c}\mathrm{CH}_{4} \text { Mass } \\
\text { Flow Rate } \\
{[\mathrm{g} / \mathrm{min}]}\end{array}$ & $\begin{array}{c}\mathrm{H}_{2} \mathrm{O} \text { Mass Flow } \\
\text { Rate } \\
{[\mathrm{g} / \mathrm{min}]}\end{array}$ & $\begin{array}{c}\text { Steam to } \\
\text { Methane } \\
\text { Ratio } \\
{[\mathrm{mol} / \mathrm{mol}]}\end{array}$ & $\begin{array}{c}\text { SMR } \\
\text { Temperature } \\
{\left[{ }^{\circ} \mathrm{F}\right]}\end{array}$ & $\begin{array}{c}\text { System } \\
\text { Pressure } \\
{[\mathrm{psia}]}\end{array}$ \\
\hline 1 & 1.637 & 5.516 & 3 & 930 & 14.7 \\
\hline 2 & 1.637 & 6.435 & 3.5 & 930 & 14.7 \\
\hline 3 & 1.637 & 7.354 & 4 & 930 & 14.7 \\
\hline
\end{tabular}




\section{SMR - Phase 2}

Observed temperature and pressure transients - Affected $\mathrm{H}_{2}$ production at certain points

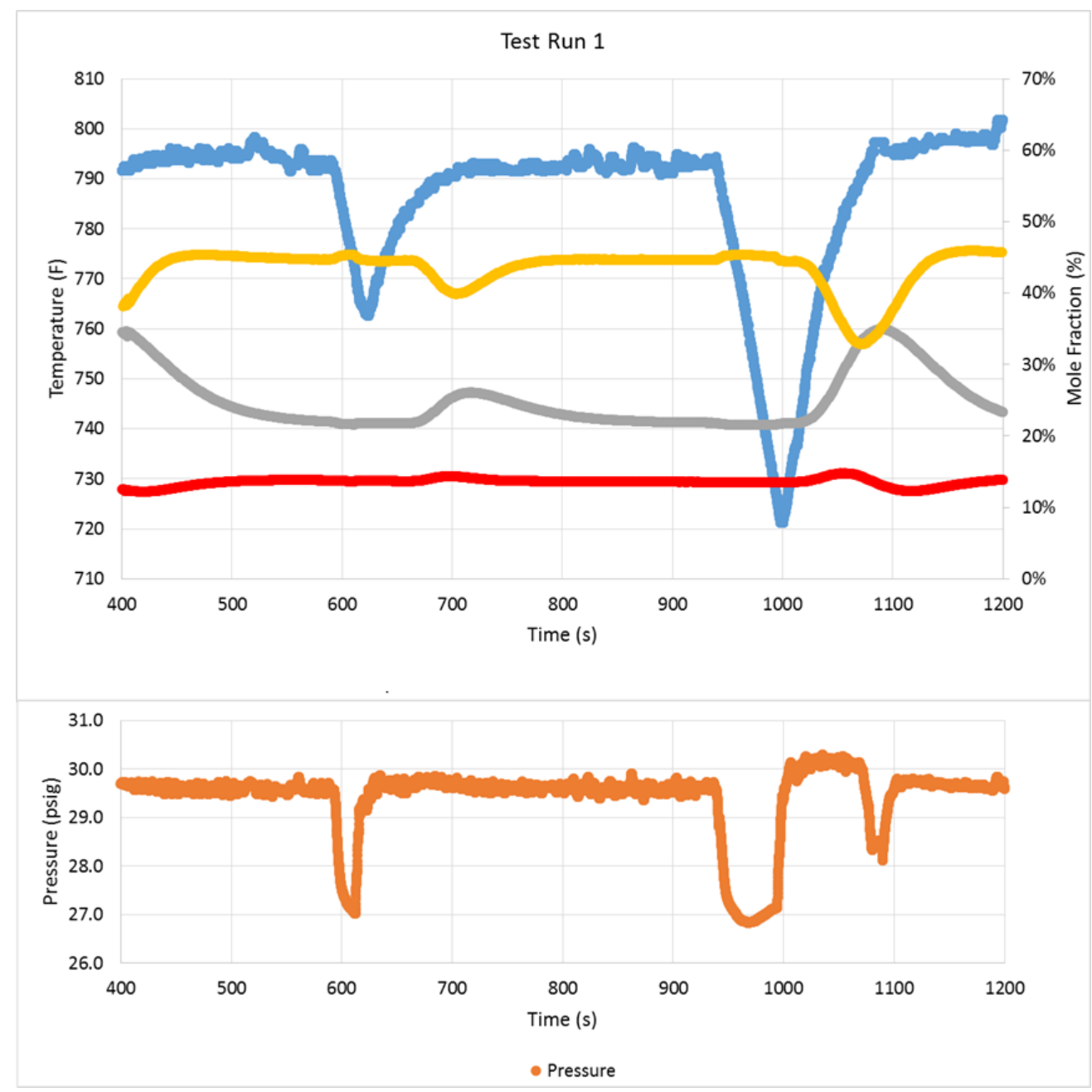

\begin{tabular}{|llll}
\hline- Temperature & $\circ \mathrm{CH} 4 \quad \mathrm{H}_{2} \quad \bullet \mathrm{CO}$ \\
\hline
\end{tabular}




\section{SMR - Phase 2}

\section{Test Results:}

- Higher overall amount of hydrogen being produced

- Carbon deposition still being generated, though at lower rate

\section{Conclusions:}

- Catalyst was not sufficiently reduced

- Need to increase thermal mass to heat up fluids to design temperatures

- Need to minimize hotspots that promote carbon deposition

- Essential to maintain consistent heating profile for input stream going into the reactor

\section{Recommendations:}

- Conduct one more round of testing, with system modifications

Carbon deposition in test system

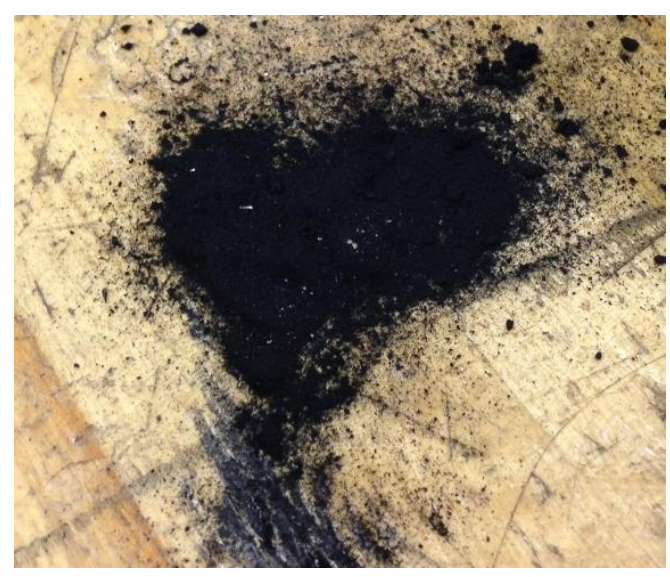




\section{SMR - Phase 3}

\section{System modifications:}

Switched to new metal foam catalyst - for increased chemical stability and potential conversion efficiency

\begin{tabular}{|c|c|c|c|c|c|c|}
\hline \multicolumn{4}{|c|}{ Heat Transfer } & \multicolumn{3}{|c|}{ Mass Transfer } \\
\hline \multicolumn{4}{|c|}{$\begin{array}{l}\text { Higher thermal conductivity } \\
\text { minimizes temperature gradients \& } \\
\text { hot spots }\end{array}$} & \multicolumn{3}{|c|}{$\begin{array}{l}\text { Porous structure provides } \\
\text { tortuous path for gas mole }\end{array}$} \\
\hline \multicolumn{4}{|c|}{$\begin{array}{l}\text { Helps favor the reactions we want } \\
\text { and prevent those we don't }\end{array}$} & \multicolumn{3}{|c|}{$\begin{array}{l}\text { Better dispersion of the ac } \\
\text { metals coated on the met } \\
\text { structure }\end{array}$} \\
\hline \multicolumn{7}{|c|}{ Hot Flow Test (With Preheating of Gases) } \\
\hline Test \# & $\begin{array}{l}\mathrm{CH}_{4} \text { Mass } \\
\text { Flow Rate } \\
\text { [g/min] }\end{array}$ & $\begin{array}{c}\mathrm{H}_{2} \mathrm{O} \text { Mass Flow } \\
\text { Rate } \\
{[\mathrm{g} / \mathrm{min}]}\end{array}$ & & $\begin{array}{l}\text { m to } \\
\text { tane } \\
\text { tio } \\
\text { mol] }\end{array}$ & $\begin{array}{c}\text { SMR } \\
\text { Temperature } \\
{\left[{ }^{\circ} \mathrm{F}\right]}\end{array}$ & $\begin{array}{c}\text { System } \\
\text { Pressure } \\
\text { [psia] }\end{array}$ \\
\hline 1 & 1.637 & 7.354 & & 4 & 930 & 14.7 \\
\hline 2 & 1.637 & 7.354 & & 4 & 1020 & 14.7 \\
\hline 3 & 1.637 & 7.354 & & 4 & 1110 & 14.7 \\
\hline 4 & 3.274 & 14.708 & & 4 & 930 & 14.7 \\
\hline 5 & 3.274 & 14.708 & & 4 & 1020 & 14.7 \\
\hline 6 & 3.274 & 14.708 & & 4 & 1110 & 14.7 \\
\hline
\end{tabular}

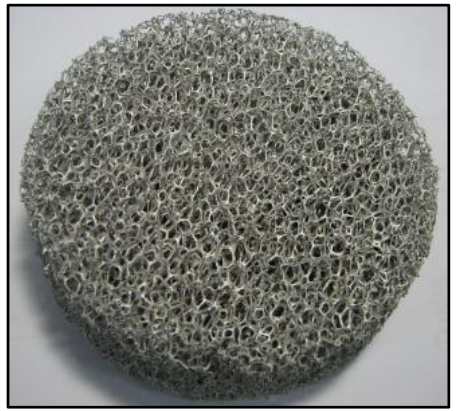

Ordered Pd/Rh coated SIC metal foam catalyst, machined to SMR physical dimensions with throughhole for high temp temperature probe 


\section{SMR - Phase 3}

\section{System modifications:}

- Installed 3-way valve downstream of steam generator, to help avoid $\mathrm{T}$ and $\mathrm{P}$ transients (circled in green)

- Installed heating tape between steam generator and reactor, to maintain consistent heating profile (circled in red)

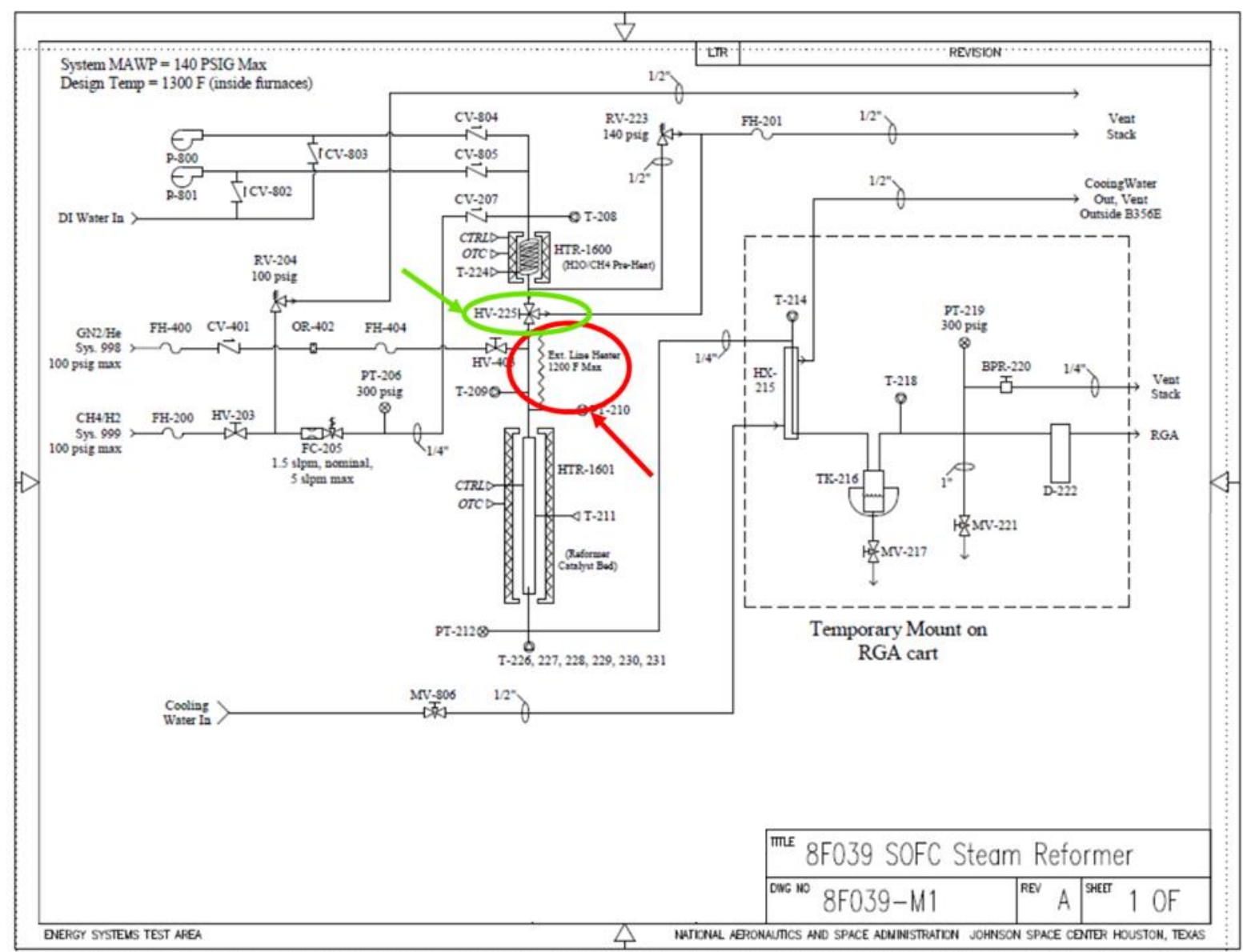




\section{SMR - Phase 3}

Initial test run - steadier temperature and pressure rates

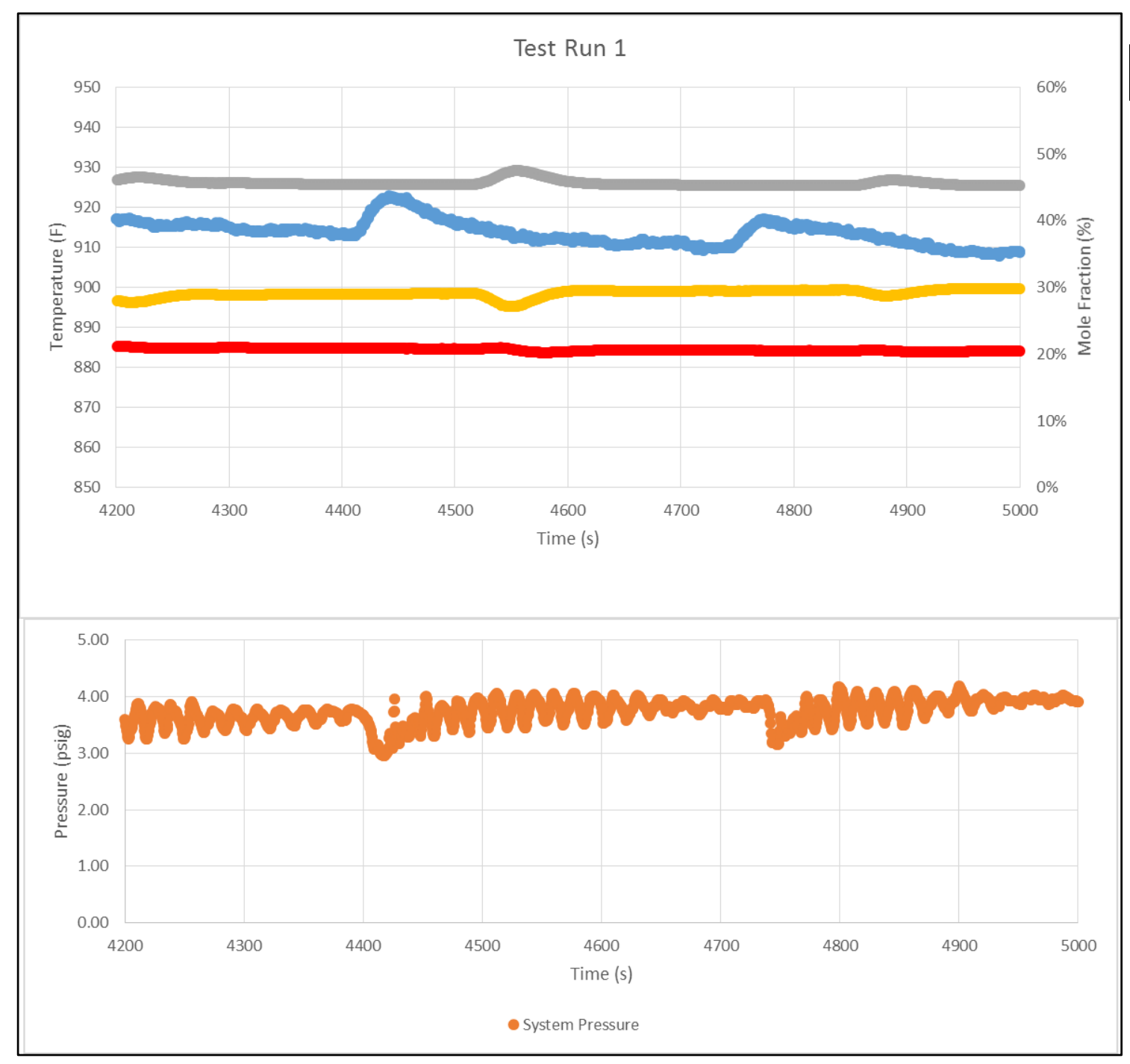




\section{SMR - Phase 3}

Final test run - steady pressure flow, fluctuating $\mathrm{H}_{2}$ production

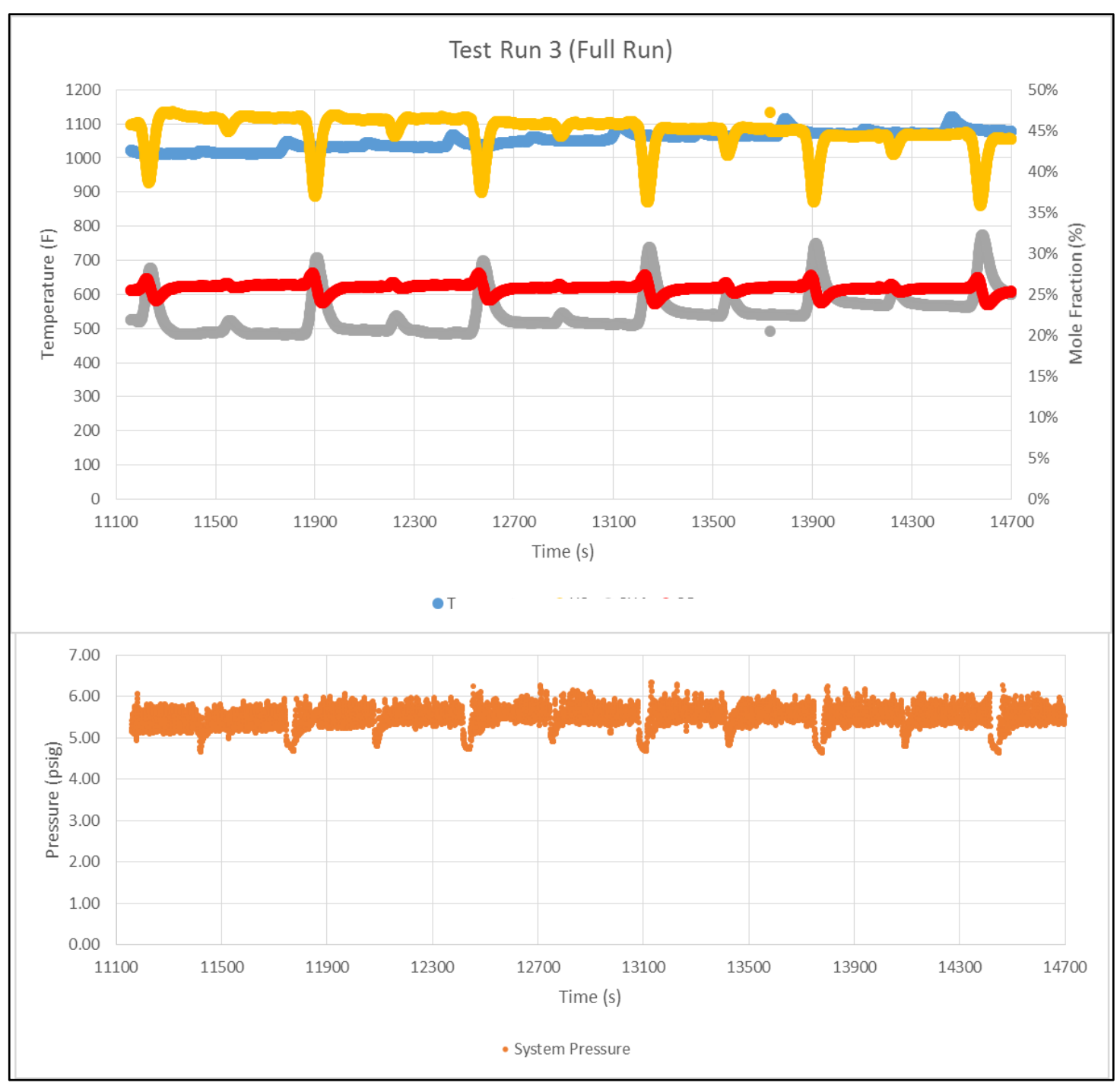

\begin{tabular}{|c|c|c|c|}
\hline & CH4 & (H) & \\
\hline
\end{tabular}




\section{SMR - Phase 3 \& Test Summary}

\section{Testing Results:}

- Test prematurely ended due to leaking relief valve

- $\quad$ Eliminated carbon deposition

- $\quad$ Similar $\mathrm{H}_{2}$ conversion rates to Phase 2

\section{Conclusions/Lessons Learned:}

- Ensure relief valve has captured venting

- When possible, run higher flow rates through new catalyst to determine whether conversion efficiency increases

- Potentially better to use Coriolis flow controller instead of syringe pumps

\section{SMR Overall Test Summary}

\begin{tabular}{|c|c|c|c|}
\hline & Phase 1 & Phase 2 & Phase 3 \\
\hline $\begin{array}{l}\text { Reformer } \\
\text { Design/Catalyst }\end{array}$ & $\begin{array}{l}4 \text { cylinder reactor/Nickel } \\
\text { Oxide Pellets }\end{array}$ & $\begin{array}{l}1 \text { cylinder reactor/Nickel } \\
\text { Oxide Pellets }\end{array}$ & $\begin{array}{l}1 \text { cylinder reactor/Pd-Rh on SIC } \\
\text { metal foam }\end{array}$ \\
\hline Test Results & $\begin{array}{l}\text {-Lower than expected } \\
\text { production of H2 (30\%-35\%) } \\
\text {-Carbon deposition blockage } \\
\text { in the catalyst bed }\end{array}$ & $\begin{array}{l}\text {-Higher H2 production } \\
(\sim 45 \%) \\
\text {-Carbon deposition generated } \\
\text { at lower rate }\end{array}$ & $\begin{array}{l}\text {-Similar } \mathrm{H}_{2} \text { conversion rates to } \\
\text { Phase } 2(\sim 45 \%) \\
\text {-Eliminated carbon deposition } \\
\text {-Test ended due to leaking relief } \\
\text { valve }\end{array}$ \\
\hline Conclusions & $\begin{array}{l}\text {-Improper steam-to-methane } \\
\text { ratio } \\
\quad \text {-No method of steam } \\
\quad \text { flow regulation } \\
\text {-Catalyst was not reduced, as it } \\
\text { should've been }\end{array}$ & $\begin{array}{l}\text {-Catalyst was not sufficiently } \\
\text { reduced } \\
\text {-Needed to increase thermal } \\
\text { mass to heat up fluids to } \\
\text { design temperatures } \\
\text {-Need to minimize hotspots }\end{array}$ & $\begin{array}{l}\text {-Ensure relief valve has captured } \\
\text { venting } \\
\text {-Run higher test flow rates through } \\
\text { new catalyst to determine whether } \\
\text { conversion efficiency increases } \\
\text {-Maybe use Coriolis flow controller }\end{array}$ \\
\hline
\end{tabular}




\section{SMR - Future Plans}

\section{Future System Integration Path}

\section{Planned Activities}

Integrated test with SOFC

Integrated test with SOFC, In-Situ

Resource Utilization (ISRU), and

$\mathrm{LOX} / \mathrm{CH}_{4}$ Cryogenic Fluid

Management (CFM)

-Feed $\mathrm{CH}_{4}$ from boiloff

\section{Time Period}

FY'16

FY'16 